\title{
Visual, proprioceptive and tactile performance in left neglect patients
}

\author{
Sylvie Chokron ${ }^{\mathrm{a}, \mathrm{b}, *}$, Pascale Colliot ${ }^{\mathrm{a}, \mathrm{d}}$, Paolo Bartolomeo ${ }^{\mathrm{c}}$, François Rhein ${ }^{\mathrm{d}}$, \\ Estelle Eusop ${ }^{\mathrm{d}}$, Philippe Vassel ${ }^{\mathrm{d}}$, Théophile Ohlmann ${ }^{\mathrm{a}}$ \\ a Laboratoire de Psychologie Experimentale, CNRS, UMR 5105, 38000 Grenoble, France \\ b Service de Neurologie, Fondation Ophtalmologique A. de Rothschild, Paris, France \\ c Département de Neurosciences cliniques, Inserm U324, Hôpital Henri Mondor, Créteil, France \\ d Centre de Rééducation et Réadaptation pour Adultes, Unité de Pathologie Cérébrale, Coubert, France
}

Received 30 January 2002; received in revised form 10 April 2002; accepted 17 April 2002

\begin{abstract} supramodal spatial bias in neglect. a proprioceptive straight-ahead pointing task while blindfolded, as well as visual and tactile bisection tasks.

In the left neglect patients, we found:

(i) no systematic deviation of the ER on the ipsilesional right side;

(ii) a significant rightward bias in visual bisection, and normal performance in tactile bisection;

(iii) no correlation among the three tasks;

(iv) that only visual bisection correlated with the severity of neglect.
\end{abstract}

Patients with unilateral spatial neglect due to right-hemisphere lesions typically fail to attend to and explore left-sided stimulus objects [32]. It has been postulated that in right-brain damaged (RBD) patients an ipsilesional displacement of the egocentric frame of reference (ER), whether visual or tactile, may be responsible for a contralesional supramodal spatial bias causing their left neglect behavior [39]. However, this hypothesis had been proposed without testing, in the same patients, the position of the ER or their performance in the visual and tactile modalities. Thus, the aim of the present study was to test the hypothesis that an ipsilateral shift of the ER is responsible for a

For this purpose, a within-subject design is required. Consequently, 12 left neglect patients and 12 control subjects were asked to perform

These results are discussed with regard to the egocentric and attentional hypothesis of neglect. (C) 2002 Elsevier Science Ltd. All rights reserved.

Keywords: Neglect; Egocentric reference; Straight-ahead pointing; Bisection

\section{Introduction}

Patients with unilateral spatial neglect due to right-hemisphere lesions typically fail to attend to and explore left-sided stimulus objects [32]. Neglect has been attributed to disordered sensory input [5], disordered internal representation of space [7], disordered attention to contralesional stimuli $[25,28,33,45,52]$ and, more recently, a disordered egocentric frame of reference (ER) [39].

Not long ago, it was postulated that the key mechanism leading to neglect is the disturbed transformation of sensory input into a supramodal ER, causing deviation of the

\footnotetext{
* Corresponding author. Tel.: +33-476-82-56-30; fax: +33-476-82-78-34.

E-mail address: chokron@ext.jussieu.fr (S. Chokron).
}

reference frame toward the side ipsilateral to the brain lesion $[39,41,64]$. This deviation, in turn, would be responsible for the impairment of these patients' performance on the left side of space in perceptual and representational tasks [39,55]. Along the same lines, it was subsequently proposed that the entire distribution of exploratory activity was shifted towards the right of the patient's sagittal body midline $[42,43]$. The authors claimed that the whole frame for exploratory behavior, whatever the modality (tactile or visual), was shifted to a new equilibrium on the right. In this hypothesis, left neglect is interpreted as a supramodal spatial bias caused by an ipsilateral deviation of the ER [43].

According to this hypothesis, left neglect signs should be of comparable severity whatever the modality. These assertions stand in sharp contrast with the literature on the position of the ER in neglect, and with the characterization of left 
neglect signs in the visual and tactile modalities. Whereas some authors have described an ipsilateral shift of the subjective sagittal middle when right-brain damaged (RBD) patients with left neglect (RBDN+) are asked to point straight ahead without any visual information [16,32,44], several authors, when testing larger groups of RBD patients with or without neglect signs (RBDN+ and RBDN- patients), have found no correlation between neglect signs in visuo-spatial tasks and either the presence or the side of a deviation of the ER position recorded during a proprioceptive straight-ahead pointing task $[1,11,13,22,47,49]$.

As regards the supramodal nature of neglect, the literature shows that when the same left neglect patients are simultaneously submitted to visual and tactile tasks, they often show a strong spatial bias in the visual task which is much less severe in the tactile modality $[26,66]$. This was the case of bisection protocols in particular. Fuji et al. [23], as well as Hjaltason et al. [34], submitted neglect patients and normal control subjects to visual and tactile bisection tasks. In the visual presentation, the rightward deviation of the objective midline was significantly more prominent in patients with visual spatial neglect than in normal controls. However, when tactually bisecting rods, there was no significant difference between the patient group and the controls in either study.

In the same way, space-exploration patterns produced under visual control and in its absence (blindfolded conditions) were compared by asking left-brain damaged (LBD) and RBD patients with and without neglect signs to press the keys of a keyboard [26]. Only RBD patients showed a preference for pressing the keys ipsilateral to the lesion, but this tendency was more marked in RBD patients with left neglect $(\mathrm{RBDN}+)$ than in RBD patients without left neglect $(\mathrm{RBDN}-)$. In the nonvisual tactile version of the test, only RBDN+ patients tended to favor the ipsilateral half of the keyboard. This ipsilateral preference was, however, significantly less marked than that found when the task was visually assisted, confirming the earlier mentioned studies using bisection protocols.

To summarize, the earlier mentioned studies indicate that neglect is often more common and more severe for visual than for nonvisual material. Recently, some of us have demonstrated that the position of the ER does not play a key role in the behavioral consequences of the spatial bias induced by right hemisphere [1]. Nevertheless, these data mainly concern the visual modality.

The aim of the present study, was to test the hypothesis that an ipsilateral shift of the ER is responsible for a supramodal spatial bias in neglect, as this hypothesis was forwarded without testing, in the same patients, the position of their ER and their performance in the visual and tactile modalities.

For this purpose, a within-subject design is required. Consequently, RBDN+ patients and control subjects were asked to perform a proprioceptive, haptic straight-ahead pointing task while blindfolded, as well as visual and tactile bisection tasks. A haptic straight-ahead pointing task was chosen for several reasons. First, in previous studies dealing with the recording of the ER position [36], the straight-ahead pointing task is done in the proprioceptive modality. Second, as one of the major aims of the present study was to study the correlation between the position of the ER and the presence of left neglect signs in the tactile modality, it seemed more adequate to use a haptic rather than a visual straight-ahead pointing task. Moreover, the comparison of the left neglect behavior between the visual and tactile modalities was already provided by the direct comparison of performance in the visual and tactile bisection tasks. According to Turvey [61], the body posture scheme is based on the perception of efforts required to move (inertia momentum) or to stabilize (static momentum) the different body segments. Consequently, the haptic straight-ahead pointing task and the ER - the main component of the body scheme-share the same haptic perceptual channel. Finally, several studies have shown both no significant difference between visual and proprioceptive straight-ahead pointing performance $[22,49]$, and a massive effect of the direction of visual motion in visual straight-ahead pointing performance that could bias the results [22]. These reasons explain our choice of a haptic, proprioceptive straight-ahead pointing task to record the position of the ER.

If, as Karnath and Perenin have proposed [43], RBDN+ patients have an ipsilesional deviation of the ER responsible for a supramodal spatial bias, one or more of the following independent consequences should be observed: (i) the average errors in straight-ahead pointing and the average errors in the subjective middle in both the visual and tactile bisection tasks $[39,42,43]$ must clearly exhibit ipsilesional deviations; (ii) whatever their mean values, these three errors must correlate with each other. For example, a strong positive correlation between straight-ahead pointing and visual bisection should occur, even if the average of the first variable is negative and that of the second is positive. Finally, we also studied the correlation between the degree of left neglect signs (assessed using a clinical battery of tests, see [4]) and the position of the ER.

\section{Subjects}

Twelve RBD + patients (mean age $=59.2$ years; S.D. $=$ 10.4 ) and 12 age-matched control subjects (mean age $=50.3$ years; S.D. $=9.2$ ) free of neurological damage consented to participate in this study. All the subjects were right handed, as assessed by means of a laterality questionnaire [21]. Table 1 summarizes the neurological and demographic data. Lesions loci were all confirmed by CT or/and MRI scans.

In the patients, the presence and severity of unilateral neglect were assessed by using a battery of visuo-spatial tests (see [4]), which included tasks of line, bell and letter A cancellations, identification of overlapping figures, a copy of the Gainotti figure, and line bisection. The direction and degree of spatial bias were estimated with the following 
Table 1

Demographic and clinical data of right-brain damaged patients, $\lambda$ scores, mean deviations (algebraic error in degrees) and standard deviations in the three tasks

\begin{tabular}{|c|c|c|c|c|c|c|c|c|c|c|c|}
\hline \multirow[t]{2}{*}{ Patient } & \multirow[t]{2}{*}{ Age } & \multirow[t]{2}{*}{ Sex } & \multirow{2}{*}{$\begin{array}{l}\text { Onset of } \\
\text { illness (days) }\end{array}$} & \multirow{2}{*}{$\begin{array}{l}\text { Locus of } \\
\text { lesion }\end{array}$} & \multirow[t]{2}{*}{ Aetiology } & \multirow{2}{*}{$\begin{array}{l}\text { Visual } \\
\text { extinction }\end{array}$} & \multirow{2}{*}{$\begin{array}{l}\text { Visual field } \\
\text { defect }\end{array}$} & \multirow[t]{2}{*}{$\lambda$ score } & \multicolumn{3}{|l|}{ Mean deviations } \\
\hline & & & & & & & & & SAP & $\mathrm{TB}$ & VMB \\
\hline $\mathrm{P \# 1}$ & 58 & M & $\cong 2000$ & $\mathrm{P}$ & Tumoral & No & LH & $\lambda=0.163$ & $+6.63^{*}(3.69)$ & $+0.69^{*}(1.19)$ & $+0.43^{*}(0.52)$ \\
\hline $\mathrm{P} \# 2$ & 71 & M & 360 & PTO & Ischemic & No & No & $\lambda=0.212$ & $+9.38^{*}(16.37)$ & $+0.19(2.26)$ & $+0.40(1.67)$ \\
\hline $\mathrm{P \# 3}$ & 77 & M & 31 & $\mathrm{P}$ & Ischemic & Yes & LH & $\lambda=1.435$ & $-0.91(17.93)$ & $+0.08(1.00)$ & $+1.63 *(0.92)$ \\
\hline P\#4 & 65 & M & 146 & PTO & Ischemic & Yes & LH & $\lambda=1.015$ & $+0.28(10.61)$ & $+0.43(1.79)$ & $+0.94^{*}(1.59)$ \\
\hline $\mathrm{P} \# 5$ & 64 & M & 44 & $\mathrm{TP}$ & Ischemic & No & LIQ & $\lambda=0.365$ & $-3.81^{*}(3.95)$ & $-0.97^{*}(0.92)$ & $+0.09(0.38)$ \\
\hline P\#6 & 48 & M & 115 & $\mathrm{~T}$ & Haemorragic & Yes & No & $\lambda=0.504$ & $-1.75(6.43)$ & $-0.94 *(1.69)$ & $+1.24 *(1.53)$ \\
\hline $\mathrm{P \# 7}$ & 65 & M & 56 & $P$ & Ischemic & No & No & $\lambda=0.135$ & $-3.31(13.33)$ & $-0.53^{*}(0.91)$ & $+0.03(0.26)$ \\
\hline P\#8 & 46 & M & 363 & FPT & Ischemic & Yes & No & $\lambda=1.011$ & $+0.38(17.74)$ & $-0.96(2.40)$ & $-0.29(1.71)$ \\
\hline P\#9 & 55 & M & 42 & FPT & Haemorragic & Yes & No & $\lambda=0.169$ & $-5.69^{*}(3.26)$ & $-0.48(3.71)$ & $+0.7^{*}(0.60)$ \\
\hline $\mathrm{P} \# 10$ & 63 & M & 85 & $\mathrm{P}$ & Ischemic & Yes & LH & $\lambda=0.964$ & $-14.8^{*}(4.38)$ & $+0.21(1.34)$ & $+0.56^{*}(0.81)$ \\
\hline $\mathrm{P} \# 11$ & 56 & $\mathrm{~F}$ & 70 & $\mathrm{TP}$ & Ischemic & Yes & No & $\lambda=0.539$ & $+6.69^{*}(9.63)$ & $-0.91^{*}(0.78)$ & $+0.69^{*}(0.27)$ \\
\hline P\#12 & 42 & $\mathrm{M}$ & 139 & $\mathrm{TP}$ & Haemorragic & Yes & No & $\lambda=0.107$ & $+5.06^{*}(2.47)$ & $+0.24(1.02)$ & $+0.15^{*}(0.25)$ \\
\hline Control group & $50.3(9.2)$ & & & & & & & & $+1.46(1.46)$ & $-0.08(0.91)$ & $+0.32(0.32)$ \\
\hline
\end{tabular}

Control group $\quad 50.3(9.2)$

F, frontal; T, temporal; P, parietal; O, occipital. Visual field defects: LH, left hemianopia; LIQ, left inferior quadranopia; SAP, straight-ahead pointing; TB, tactile bisection; VMB, visuo-motor bisection. The asterisk indicates a significant deviation compared to the objective middle. 
formula:

$\lambda=\ln \left(\frac{\mathrm{XR}}{\mathrm{XL}}\right)$

XR was computed by adding the number of items canceled or copied on the right half of the page, the number of items identified on the right side of the overlapping figures test, and the distance between the left end and the subjective middle of the line in the bisection task. XL was similarly computed (i.e. by adding the number of left-sided canceled items to the number of left-side copied items, the number of superimposed figures identified on the left side, and the distance between the right end and the subjective middle of the line in the bisection task).

Patients were considered to have left neglect and were thus included in this study if their $\lambda$ score exceeded the cutoff score defined as the mean +3 S.D. of $y$ scores in 30 control subjects (i.e. +0.104 ; for details see [4]).

\section{Methods and results}

\subsection{Experiment 1: straight-ahead pointing}

\subsubsection{Procedure}

Subjects were seated blindfolded in front of a large graduated table (see Fig. 1). Their trunk and head were aligned at $0^{\circ}$, the sagittal middle corresponding to the objective center of the table. Trunk and head positions were carefully monitored by an experimenter throughout the task.

Subjects were asked to point straight ahead with their right hand while blindfolded. They performed 16 trials, four for each of the four starting positions, i.e. $30^{\circ}$ to the left of the objective middle, $15^{\circ}$ to the left, $30^{\circ}$ to the right of the objective middle and $15^{\circ}$ to the right. Before each trial, the subject's arm was positioned at one of these starting points, from which they had to point straight ahead, moving the arm along the table; the index fingertip was always in contact with the table (see [16]). There was no time limit and the finger position was recorded when the subject judged

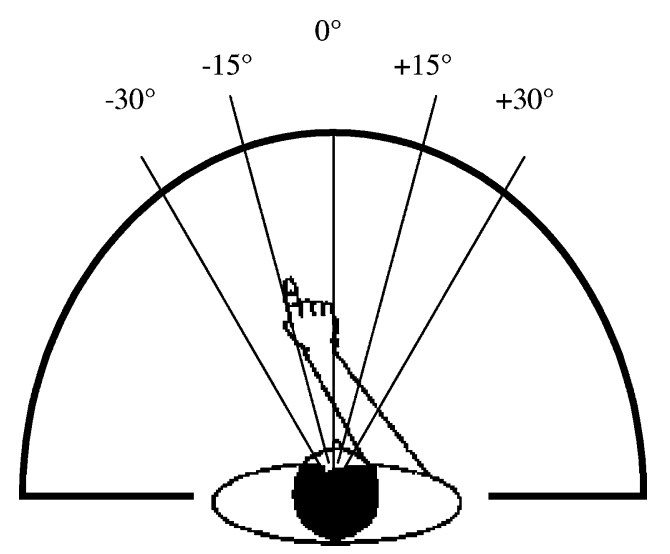

Fig. 1. Straight ahead pointing protocol. that his/her index was pointing straight ahead. The pointing error was measured to within half a degree, by determining the distance between the pointing position and the objective center, and carried a minus sign for leftward pointing and a plus sign for rightward pointing.

\subsubsection{Results and discussion}

3.1.2.1. Control subjects. The control subjects tended to point slightly to the right of the objective sagittal middle with their right hand $\left(m=+1.46^{\circ}\right.$; S.D. $=7.67 ; t(11)=$ $0.66 ; P>0.52)$, confirming previous results $[1,10,11,13]$ (see Fig. 2 and Table 2). The starting point had no significant effect in the control subjects, as previously reported [13].

3.1.2.2. Neglect patients. When asked to point straight ahead, the left neglect patients tended to deviate to the left of their objective sagittal middle $(m=-0.15$; S.D. $=$ 12.24; see Fig. 2 and Table 2). This deviation did not reach significance, when compared to the objective middle $(t(11)=0.04 ; P>0.96)$ or to the controls' performance $(t(11)=0.39 ; P>0.70)$. Contrary to the control subjects, the position of the starting point significantly influenced the position of the subjective sagittal middle in the left neglect patients $(F(3,3)=7.30 ; P<0.0007)$. The more leftward the starting point, the more the pointing was deviated to the left; likewise, the more rightward the starting point, the more the pointing was deviated to the right (see Table 2), confirming previous results [1,11-13].

In keeping with previous findings $[1,11,13,22,47]$ when pointing straight ahead, five of the 12 neglect patients

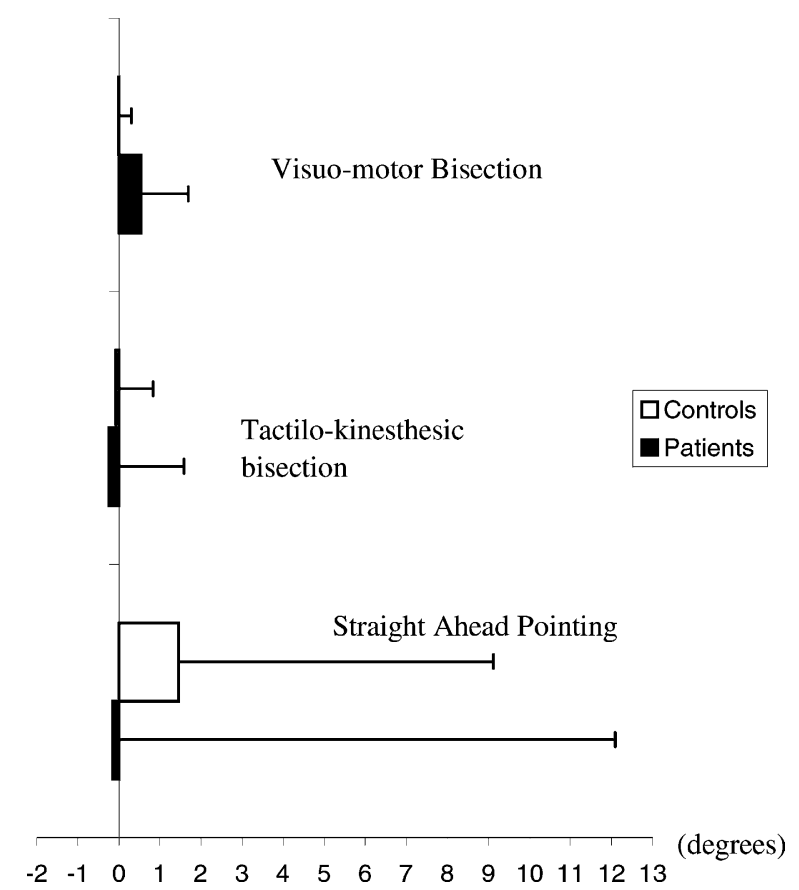

Fig. 2. Straight ahead pointing, tactilo-kinesthetic bisection and visuomotor bisection in control subjects and left neglect patients (mean deviation in degrees: - , leftward deviation; + , rightward deviation). 
Table 2

Straight-ahead pointing task: mean constant errors (algebraic error in degrees) and standard deviations

\begin{tabular}{|c|c|c|c|c|}
\hline & \multicolumn{2}{|l|}{ Left SP } & \multicolumn{2}{|l|}{ Right SP } \\
\hline & $-30^{\circ}$ & $-15^{\circ}$ & $+15^{\circ}$ & $+30^{\circ}$ \\
\hline P\#1 & $+4(3.14)$ & $+8.5(4.8)$ & $+4.63(1.11)$ & $+9.38(2.13)$ \\
\hline $\mathrm{P \# 2}$ & $-13(8.6)$ & $+7.5(9.33)$ & $+15.5(0.58)$ & $+27.50(4.36)$ \\
\hline P\#3 & $-23.25(3.5)$ & $-10.75(1.89)$ & $+9.88(2.32)$ & $+20.50(5.2)$ \\
\hline P\#4 & $-8.88(6.12)$ & $-4.63(2.29)$ & $+6.63(3.09)$ & $+8(15.3)$ \\
\hline P\#5 & $-3.75(5.25)$ & $-5.25(4.11)$ & $-2.75(3.20)$ & $+3.50(4.36)$ \\
\hline P\#6 & $-1.25(4.57)$ & $+4.75(7.41)$ & $-5(5.94)$ & $-5.50(2.52)$ \\
\hline P\#7 & $-17.88(6.3)$ & $-12(5.6)$ & $+5.13(5.39)$ & $+11.50(4.20)$ \\
\hline P\#8 & $-19.25(3.2)$ & $-11.13(3.42)$ & $+8.75(7.27)$ & $+23.13(5.17)$ \\
\hline P\#9 & $-7.75(1.5)$ & $-6.50(2.38)$ & $-4(4.08)$ & $-4.50(4.04)$ \\
\hline P\#10 & $-16.25(3.77)$ & $-14(1.41)$ & $-14.50(4.93)$ & $-14.25(7.18)$ \\
\hline P\#11 & $+4.75(9.64)$ & $+2.25(4.35)$ & +11.50 (8.39) & $+8.25(14.73)$ \\
\hline P\#12 & $+4.63(2.69$ & $+6.75(2.5)$ & $+4.63(1.89)$ & $+4.25(2.87)$ \\
\hline Patient group & $-8.16(10.68)$ & $-2.88(9.1)$ & $+3.36(9.12)$ & $+7.06(13.79)$ \\
\hline Control group & $-0.67(8.33)$ & $+0.83(7.41)$ & $+2.04(6.87)$ & $+3.65(7.57)$ \\
\hline
\end{tabular}

A left deviation is coded as '-'; a right deviation is coded as '+'; SP, starting position.

(patients \#3, \#4, \#6, \#7 and \#8) showed no significant bias, three patients (patients \#5, \#9 and \#10) deviated significantly to the left, and four patients (patients \#1, \#2, \#11 and \#12) deviated significantly to the right. Far from confirming a systematic ipsilesional shift of the ER in RBD patients with left neglect $[38,39,43]$, these findings are consistent with those of recent studies showing that the position of the ER does not correlate with the presence of left neglect signs $[11,12,22,49]$.

Moreover, only two patients (\#1 and \#12) exhibited systematic right-shifted pointing whatever the starting point (left-sided versus right-sided). In six patients (\#2, \#3, \#4, \#6, \#7 and \#8), the scanning direction induced a deviation of the subjective sagittal middle towards the starting point (rightward deviation for right-to-left scanning, and leftward deviation for left-to-right scanning), confirming the results of studies using the same protocol [11-13]. In patients \#3, $\# 4$, \#7 and \#8, the absence of a significant overall deviation stemmed from the opposite deviations that occurred with a rightward versus a leftward motor direction (see Table 2).

This effect of the starting point on the position of the ER in left neglect patients has also been found in the visual [22] and auditory [63] modalities. These two studies clearly demonstrated that, in left neglect patients, deviation of the ER was dependent on and embedded in the (visual or auditive) scanning direction. Here we confirm the results of Farne et al. [22], by showing that in four out of 12 left neglect patients, changing the side of the starting point (left versus right) is enough to abolish the deviation of the ER.

\subsection{Experiment 2: tactile bisection}

\subsubsection{Procedure}

3.2.1.1. Stimuli. Two rods $5 \mathrm{~mm}$ in diameter, fixed to a wooden support and placed on a table bearing a mark corresponding to the sagittal middle of the subject. This is an adaptation of apparatus used in previous studies $[8,15,58,59]$. The rods were 10 and $22 \mathrm{~cm}$ in length.

3.2.1.2. Method. The stimuli were presented in the horizontal plane. The rod was centered with respect to the sagittal middle of the subject's trunk. Subjects were blindfolded and the test began when the experimenter placed the subject's index finger at one extremity of the rod. After rod exploration (back and forth), the subject was asked to stop at a point that he/she estimated to be the middle of the rod. For example, when starting from the left end of the rod, the subject was asked to do a left-to-right exploration, then to come back to the left end and to identify the subjective middle while exploring the rod from left to right. In this way, when the starting point was on the left side, the last exploration of the rod took place from left to right, and vice versa. Each subject performed 16 trials, with the right hand. Each block was divided into eight trials starting from the right end and eight starting from the left end of the rod. For each block the order of presentation of the two rods of different lengths, and the starting position (left or right end), were drawn at random. All the conditions were chosen at random and the sequence differed from one subject to another. Thus, data were collected for each condition according to the length of the rod and the starting point.

The error was measured to the nearest millimeter by determining the distance between the subjective middle and the objective middle of the rod (corresponding to the sagittal middle of the subject and to $0^{\circ}$ ). A rightward deviation of the subjective middle carried a plus sign, and a leftward deviation carried a minus sign.

\subsubsection{Results and discussion}

3.2.2.1. Control subjects. While bisecting the rods with their right hand, the control subjects showed a nonsignificant leftward deviation $\left(m=-0.08^{\circ}\right.$; S.D. $=0.91 ; t(11)=$ 
Table 3

Tactile bisection task: mean deviations (algebraic error in degrees) and standard deviations

\begin{tabular}{|c|c|c|c|c|}
\hline & \multicolumn{2}{|l|}{$10 \mathrm{~cm}$ line } & \multicolumn{2}{|l|}{$20 \mathrm{~cm}$ line } \\
\hline & Left SP & Right SP & Left SP & Right SP \\
\hline P\#1 & $+0.63(0.30)$ & $+0.08(0.44)$ & +2.20 (1.49) & $-0.13(0.39)$ \\
\hline $\mathrm{P \# 2}$ & $+0.75(0.77)$ & $-0.33(0.76)$ & $-2.65(0.83)$ & $+3.00(1.35)$ \\
\hline P\#3 & $+0.83(0.62)$ & $-1.20(0.68)$ & $-0.08(0.29)$ & $+0.78(0.72)$ \\
\hline P\#4 & $-0.13(0.75)$ & $-0.23(0.45)$ & $-1.18(0.24)$ & $+3.25(0.49)$ \\
\hline $\mathrm{P \# 5}$ & $-0.80(0.73)$ & $-0.75(0.71)$ & $-0.75(0.29)$ & $-1.58(1.58)$ \\
\hline P\#6 & $+0.65(1.08)$ & $-2.70(1.50)$ & $+0.05(0.58)$ & $-1.78(0.93)$ \\
\hline P\#7 & $+0.18(0.57)$ & $-0.75(0.21)$ & $-1.25(1.20)$ & $-0.30(0.94)$ \\
\hline P\#8 & $+1(0.88)$ & $-2.33(1.05)$ & $-1.18(0.29)$ & $-1.33(4.38)$ \\
\hline P\#9 & $+2.75(3.77)$ & $-2.58(3.03)$ & +0.08 (4.27) & $-2.18(1.84)$ \\
\hline P\#10 & $+0.88(1.65)$ & $-1.38(0.48)$ & $+0.90(0.68)$ & $+0.43(0.99)$ \\
\hline P\#11 & $-0.88(0.60)$ & $-0.73(0.53)$ & $-1.58(0.96)$ & $-0.48(0.78)$ \\
\hline $\mathrm{P} \# 12$ & $+1.2(0.24)$ & $0.00(0.16)$ & $-0.63(1.49)$ & $+0.38(0.75)$ \\
\hline Patient group & $+0.59(1.50)$ & $-1.07(1.37)$ & $-0.50(1.79)$ & $+0.01(2.20)$ \\
\hline Control group & $+0.19(0.56)$ & $-0.34(0.57)$ & $+0.11(1.04)$ & $-0.27(1.19)$ \\
\hline
\end{tabular}

A left deviation is coded as '-'; a right deviation is coded as '+' SP, starting position.

0.29; $P>0.77$; see Fig. 2 and Table 3), confirming the "pseudoneglect" phenomenon originally described by Hjaltason et al. [34]. In accordance with previous studies [15], the length of the rod did not significantly affect the subjects' performance (see Table 3).

We found no significant effect of the starting position (left or right end) or, thus, of the scanning direction prior to bisection. This contrasts with results obtained by Philip and Hatwell [48], who reported that control subjects scanning a rod from left to right made a rightward deviation, and vice versa. However, in their study, the subject's finger was always positioned at the left extremity of the rod, and the subjects scanned from left to right as many times as was necessary to bisect the rod. In contrast, in our study, when the subject started at the left end, he/she could only explore the rod once from left to right, then once from right to left, and then had to bisect the rod during the last left to right scan. Although this methodological point might account for the different findings, published results on bisection by control subjects are remarkably variable and inconsistent (for review see [37]).

3.2.2.2. Neglect patients. When asked to place their right index on the subjective middle of a tactually perceived rod, the left neglect patients erred to the left of the objective middle $\left(m=-0.25^{\circ} ;\right.$ S.D. $=1.84$; see Table 3$)$. This deviation did not differ significantly either from the objective middle $(t(11)=0.46 ; P>0.65)$ or from the mean control value $\left(m=-0.08^{\circ} ;\right.$ S.D. $\left.=0.91 ; t(11)=0.29 ; P>0.77\right)$.

The length of the rod significantly interacted with the side of the starting point (left or right) $(F(1,7)=5.37 ; P<$ $0.5)$. With the smallest rod, the left starting point induced a rightward deviation, and vice versa (see Table 3 ). In contrast, with the longer rod the subjective middle was deviated to the side of the starting point (see Table 3). Hjaltason et al. [34] also found that, among patients with RBD, only those with left neglect signs deviated to the side of the starting point when bisecting rods of 20 and $40 \mathrm{~cm}$.

When tactually bisecting the rods, four of the 12 neglect patients presented a significant leftward deviation (patients \#5, \#6, \#7 and \#11), and only one neglect patient (\#1) exhibited a significant rightward deviation. However, this rightward deviation did not differ significantly from that observed in the controls $\left(m=+0.69^{\circ}\right.$; S.D. $=1.19 ; t(15)=2.06$; $P>0.05)$; in addition, when this patient's results were analyzed separately for each starting point, the right-hand starting point induced a more marked rightward deviation than did the left-hand starting point (see Table 3). Six of the 12 patients did not deviate significantly from the objective middle of the rod, confirming that, contrary to visual line bisection, left neglect patients perform in the same way as controls in tactile bisection protocols [23,24].

\subsection{Experiment 3: visuo-motor bisection}

\subsubsection{Procedure}

3.3.1.1. Stimuli. Two black lines 10 and $22 \mathrm{~cm}$ in length (same length as the rods). Each line was presented centered on a separate $29.7 \mathrm{~cm} \times 21 \mathrm{~cm}$ sheet of paper.

3.3.1.2. Method. The stimuli were presented in the horizontal plane at a distance of approximately $50 \mathrm{~cm}$ from the subject. The line was centered with respect to the sagittal middle of the subject's trunk. The middle of the sheet corresponded to the objective middle of the line and to the actual sagittal middle of the subject. The test began when the experimenter placed the sheet on the table. There was no time limit. The trial stopped when the subject placed a pencil mark at the point he/she judged to be the middle. Only the right hand was used. The two lines of different lengths were presented at random. The subject performed 
16 trials: eight with the $10 \mathrm{~cm}$ line and eight with the $22 \mathrm{~cm}$ line.

The error was measured to the nearest millimeter by determining the distance between the subjective middle and the objective middle of the line (corresponding to the sagittal middle of the subject and to $0^{\circ}$ ). A rightward deviation of the subjective middle carried a plus sign, and a leftward deviation carried a minus sign.

\subsubsection{Results and discussion}

3.3.2.1. Control subjects. When bisecting the lines (with their right hand), the control subjects tended to place the subjective middle to the left of the objective middle ( $m=$ $-0.004^{\circ}$; S.D. $=0.32 ; t(11)=0.04 ; P>0.96$; see Table 4). This finding is consistent both with previous results $[23,26,31,34,49,66]$ and with the "pseudoneglect" phenomenon originally described by Bowers and Heilman [8]. The length of the line did not significantly affect the position of the subjective middle (see Table 4).

3.3.2.2. Neglect patients. When the left neglect patients visually bisected the lines, they tended to made a rightward deviation $\left(m=+0.55^{\circ}\right.$; S.D. $\left.=1.14\right)$, although the difference was not significant relative to the objective middle $(t(11)=1.66 ; P>0.12)$ or to the control values $(m=$ $-0.003^{\circ}$; S.D. $\left.=0.32 ; t(11)=1.86 ; P>0.08\right)$. The length of the line had a significant effect on the subject's bias direction $(F(1,7)=9.70 ; P<0.02)$, with a leftward deviation on the shorter line $\left(m=-0.002^{\circ} ;\right.$ S.D. $=0.86 ; t(11)=$ $0.007 ; P>0.99 \mathrm{~ns})$ and a significant rightward deviation on the longer line $\left(\left(m=+1.1^{\circ} ;\right.\right.$ S.D. $=1.13 ; t(11)=3.37$; $P<0.01$; see Table 4). This length effect, known as the "cross-over" effect [31,60], may account for the absence of a significant overall deviation in the neglect patients.

As shown in Table 4, only one of the 12 neglect patients (patient \#8) exhibited a leftward, albeit nonsignificant, devi-

Table 4

Visuo-motor bisection task: mean deviations (algebraic error in degrees) and standard deviations

\begin{tabular}{lll}
\hline & $10 \mathrm{~cm}$ line & $20 \mathrm{~cm}$ line \\
\hline P\#1 & $+0.42(0.56)$ & $+0.43(0.52)$ \\
P\#2 & $-0.71(0.74)$ & $+1.51(1.61)$ \\
P\#3 & $+0.84(0.3)$ & $+2.42(0.54)$ \\
P\#4 & $-0.39(1)$ & $+2.26(0.65)$ \\
P\#5 & $-0.06(0.19)$ & $+0.24(0.47)$ \\
P\#6 & $+0.55(1.03)$ & $+1.94(1.71)$ \\
P\#7 & $+0.16(0.18)$ & $-0.11(0.26)$ \\
P\#8 & $-1.76(0.6)$ & $+1.18(1)$ \\
P\#9 & $+0.43(0.41)$ & $+0.98(0.65)$ \\
P\#10 & $-0.08(0.37)$ & $+1.2(0.58)$ \\
P\#11 & $+0.50(0.09)$ & $+0.89(0.24)$ \\
P\#12 & $+0.08(0.18)$ & $+0.22(0.3)$ \\
Patient group & $0.00(-0.86)$ & $+1.1(1.13)$ \\
Control group & $-0.01(0.18)$ & $-0.002(0.41)$ \\
\hline A & $6-20 f$ reviation &
\end{tabular}

A left deviation is coded as '-'; a right deviation is coded as ' + '. ation. The other 11 left neglect patients bisected to the right of the objective middle. This rightward bias was significant in eight patients relative to the objective middle and in seven patients relative to the control values. This confirms reports that visual line bisection protocols are sensitive to left neglect signs [9,23,26,34].

One patient (\#2) showed a rightward deviation, but this was not significant, probably owing to the variability of the patterns obtained between the two line lengths. A cross-over effect $[31,60]$ was observed in four cases (patients \#2, \#4, $\# 8$ and \#10): the side of the observed error depended on the length of the line (leftward bias with the $10 \mathrm{~cm}$ line, significant rightward bias with the $22 \mathrm{~cm}$ line).

\subsection{Correlations}

A one-tailed significance level of 0.05 was adopted for all the analyses. Given that rejection of $\mathrm{H} 1$ does not necessarily correspond to acceptation of $\mathrm{H} 0$, it is necessary to take the "effect sizes" into account $[17,18,56]$. As regards Bravais-Pearson correlational studies, Corroyer and Rouanet [19] reported that effect sizes lower than the benchmark value of 0.30 correspond to a "weak effect". Only in this case does the acceptation of $\mathrm{H} 0$ become plausible, when $\mathrm{H} 1$ is rejected.

In accordance with the hypothesis of a signed deviation from zero for each of the variables, correlations will be computed from the algebraic values. If the ER, in the straight-ahead pointing task, acts as a factor of general deviation, positive correlations are expected. Thus, the more the patient deviates toward a given side for a given variable, the more he/she will deviate toward the same side for the other variables.

\subsubsection{Comparison between straight-ahead pointing, tactile bisection and visual bisection task performances}

Correlations obtained with algebraic value are reported in Table 5. No links between the three variables were found in the left neglect patients: the direction and size of errors in one task were independent from the direction and size of errors in another task. All the effects were weak or nonexistent $(<0.30$, Corroyer and Rouanet's benchmark). A positive correlation was observed between the straight-ahead pointing and tactile bisection performances of the controls; these two tasks could share a common variance stemming from haptic activity.

Table 5

Correlation between the three tasks for control subjects and left neglect patients

\begin{tabular}{llll}
\hline & Controls & Left neglect patients $(\mathrm{ns})$ & $\chi^{2}(\mathrm{~ns})$ \\
\hline SAP/TB & $r=0.495, P=0.10$ & $r=0.169$ & 0.649 \\
SAP/VB & $r=-0.016 \mathrm{~ns}$ & $r=-0.083$ & 0.016 \\
TB/VB & $r=-0.116 \mathrm{~ns}$ & $r=0.194$ & 0.036 \\
\hline
\end{tabular}

SAP, straight-ahead pointing; TB, tactile bisection; VB, visual bisection. 
Table 6

Comparison between the three tasks (in terms of algebraic error and absolute error) and the severity of USN ( $\lambda$ score)

\begin{tabular}{lll}
\hline$\lambda$ score & Straight ahead & Tactile bisection (ns) \\
\hline Algebraic error & $r=-0.317, P<0.10$ & $r=0.039$ \\
Absolute error & $r=0.542, P=0.10$ & $r=-0.073$ \\
\hline
\end{tabular}

The Fisher comparison between the two correlations of each line (Table 5) showed no significant difference between the control and patient groups.

It is important to check that the straight-ahead pointing deviation acts as a factor on the correlation between tactile and visual bisection performance. The partial correlation coefficients between these two bisections, with the straight-ahead pointing deviation held constant, for the patient and the control group, were respectively 0.211 (.194 without removing egocentric deviation) and -0.124 ( -0.116 without removing egocentric deviation). Consequently, given the quasi equality of the two statistics, the link between the two bisections was independent of straight-ahead pointing performance.

Finally, it is important to check that the USN (Unilateral Spatial Neglect) score determined with a battery of visuo-spatial tasks (see [28]) acts as a factor on the correlation between each task. With the USN score held constant, the partial correlation coefficient between the straight-ahead pointing task and the visual bisection task was 0.08 ( -0.083 without removing the USN score), that between the straight-ahead pointing task and tactile bisection was 0.191 ( 0.169 without removing the USN score) and that between the visual and tactile bisection tasks was 0.198 (0.194 without removing the USN score). Consequently, considering the quasi equality of the two statistics, the absence of the link between the three tasks was independent of the neglect score.

\subsubsection{Comparison between the severity of USN and straight-ahead pointing performance}

Left neglect signs are assessed by a battery of clinical tests from which an index is computed. This index increases with the severity of the USN. For this reason, the egocentric hypothesis of neglect predicts that it should mainly correlate with straight-ahead pointing performance. On the basis of algebraic values (Table 6), the USN showed a strong tendency to correlate positively with visual bisection $(0.466)$. The existence of a negative link ( -0.317$)$ with straight-ahead pointing cannot be discarded, because of the "effect size" (above the benchmark).

Finally, tactile bisection was independent from USN severity (0.039). The absolute values confirmed these results (see Table 6): errors, whatever their direction, in straight-ahead pointing $(0.542)$ and in visual bisection (0.697) correlated positively with the USN score. A visual bisection task is included in the USN score, so the link between visual bisection and the USN score is trivial, simply confirming the reliability of this kind of task. On the other hand, the actual links between straight-ahead pointing and the NSU score are problematic. The more severe the USN, the larger the absolute errors but the smaller the algebraic error. Considering egocentric hypotheses of neglect, it is important to examine whether the ER position recorded during the straight-ahead pointing task is responsible for the significant link observed between visual bisection performance and neglect signs. With straight-ahead pointing deviation held constant, the partial correlation coefficients between visual bisection and the USN score were unchanged (partial correlations of algebraic and absolute values: 0.465 and 0.581 , respectively). Thus, straight-ahead pointing position cannot explain the observed link between the severity of neglect and the visual bisection bias.

\section{General discussion}

The aim of this study, was to test the hypothesis that an ipsilesional deviation of the ER is responsible for a rightward shift of the entire distribution of exploratory activity, whatever the modality $[39,42,43]$. For this purpose, RBDN+ patients and controls were asked to perform a proprioceptive straight-ahead pointing task while blindfolded, as well as visual and tactile bisection tasks.

First of all, before discussing the working hypothesis of a correlation between the position of the ER, the tactile and the visual bisection performance, we confirmed previous data obtained in normal subjects and neglect patients when performing these three tasks. Indeed, we showed that left neglect patients do not have a systematic ipsilesional shift of their haptic ER [1,11,13,22,47]. Also confirming previous results [23,34], while the visual line-bisection protocol showed a significant rightward bias in eight of the 12 patients, tactile rod bisection performance did not differ in normal and left neglect patients, taking the form of an overall, nonsignificant leftward deviation of the subjective middle in both groups.

Questioning the working hypothesis, no correlation was found among the three tasks in left neglect patients, whereas a correlation emerged in the control group between performance in the straight-ahead pointing task and the tactile bisection task. These findings are discussed later on.

\subsection{Absence of a systematic ipsilesional deviation of the egocentric reference in left neglect patients}

In accordance with some previous studies [1,11,13,22,47], but contradicting others $[36,38,39,43]$, we found that 
unilateral spatial neglect was not associated in RBD patients with a systematic ipsilesional shift of the ER.

At the very most, we found a link between the severity of left neglect and the size of the deviation (in terms of precision). This confirms the recent study by Pizzamiglio et al. [49], who also reported that "despite the lack of any systematic shift of body midline perception, neglect patients' performance in the two midline tasks (visual and proprioceptive) were far from normal" (p. 480). These authors clearly showed that the neglect patients' perceptual judgments were less precise, with frequent judgment errors over a wider region of space around their subjective body midline. As proposed by Pizzamiglio et al. [49] a distortion of the ER more complex than a simple ipsilesional deviation may be present in left neglect patients.

In addition, we confirm that the position of the ER is dependent on the scanning direction used by RBD patients with left neglect to reach the subjective sagittal midline position. As Farne and coworkers have pointed out [22], when the scanning direction is controlled, left neglect patients may no longer manifest any ipsilesional deviation of their ER. This rules out any causal relationship between deviation of the ER and clinical signs of neglect, but underlines the role of scanning strategies on the amount of left neglect signs, as demonstrated in other visuo-spatial protocols, including visual [22] and auditory straight-ahead pointing [65], line bisection [14,46,53] and rod bisection [34]. More experiments are needed to assess the nature of this effect of the scanning direction on estimation of the subjective middle, which, according to Halligan et al. [29], could be interpreted in terms of the direction of "the attentional spotlight used to approach the middle." Asking left neglect patients to scan from left to right, as normal left-to-right (right handed) readers usually do [14], may restore normal distribution of attention along the scanpath.

\subsection{Absence of significant spatial bias in normal and neglect patients in the tactile bisection task}

The nonsignificant spatial bias during tactile bisection by RBD patients with left neglect confirms some previous studies designed to compare visual and tactile left neglect. In fact, the few studies designed to investigate tactile neglect have yielded contradictory results. Fuji et al. [23] failed to demonstrate any tactile neglect in RBD patients with visual spatial neglect and suggested that, at least in a line bisection task, this deficit is modality specific. This agrees with data from Hjaltason et al. [34], who asked neglect patients to perform a visual, a visuo-tactile and a tactile bisection task. They observed larger rightward errors in the visual conditions but no significant deviation from the actual midpoint in the visuo-tactile or tactile conditions.

Chedru [9] designed a test suitable for presentation in equivalent tactile and visual versions: subjects were required, with and without a blindfold, to tap the keys all over a teletype keyboard as quickly as possible. RBD patients with visual field defects showed no impairment in tapping the left-sided keys when vision was obscured, while they preferred the right-sided keys when visual control was available. Chedru's conclusion was that the unilateral defect in a manual exploration of space is induced by vision. Using the same protocol, Gentilini and coworkers [26] reported that RBD patients with neglect preferred the right-sided keys, both with and without visual control, although this ipsilateral preference was significantly less marked in the tactile conditions. Our results are also consistent with Villardita's report that patients with left visual spatial neglect do not have impaired tactile exploration on the left side [66].

This suggests that vision, including head turning and eyeball movement, plays a role in unilateral spatial neglect. However, it is at variance with other data $[6,20,43,67]$ showing an impairment in the tactile exploration of contralateral space in RBD patients.

According to Gentilini and coworkers [26], the increase in ipsilateral responses when key pressing was guided by vision in comparison to the blindfolded condition suggests that incoming sensory stimuli from the ipsilateral side play a role in shifting attention towards it and in enhancing neglect of controlateral space. In fact, although several experiments have shown that attention can be allocated to different parts of the spatial field without overt eye movements (for review see [51]), experiments performed in normals have suggested that eye movements cannot be made without shifting the focus of attention in the same direction (for review see [24]).

If neglect behavior results not only from hypoattention to contralateral stimuli but also from hyperattention to ipsilateral stimuli, it is conceivable that a task carried out in the absence of sensory stimulation, such as the tactile test, entails a less marked imbalance between the two halves of space than the same task carried out with visual assistance. This suggests that visually presented stimuli may exacerbate neglect [35]. In contrast, a shift of the ER, as proposed by Karnath and coworkers $[38,39,43]$, would be unlikely to express itself more in the visual than in the tactile space.

Although, it seems reasonable to think in terms of less rightward attraction in the tactile than in the visual conditions of bisection, the two protocols are not strictly equivalent. Indeed, the performance of neglect patients in the two tests may be influenced not only by visual information but also by different exploration strategies, such as head and eye movements and motor programs. These conflicting results are likely attributable to task differences among the reported studies. In visual spatial neglect, many subjects have unilateral spatial neglect in some but not all tasks involving reading, searching, line bisection, drawing, etc. (see [30]). Therefore, the spatial neglect phenomenon may be task dependent, and, in the tactile modality, the line bisection task may fail to reveal unilateral neglect. 


\subsection{Absence of correlation between the position of the egocentric reference and visual and tactile bisection performance}

Karnath [40] proposed that "the whole frame for exploratory behavior is shifted to a new equilibrium on the right." On the basis of this deviation model, Karnath and Perenin [43] suggested that "the character of this frame appears to be supramodal in that it determines the distribution of exploratory movements irrespective of whether the subject explores the surround visually or by touch." In this model, a significant correlation should be observed between proprioceptive straight-ahead pointing, tactile and visual bisection performance. However, the findings reported here further support a dissociation between left neglect signs and the position of the ER recorded in the visual or proprioceptive modality. Recently, Pizzamiglio et al. [49] submitted ten RBD patients with left unilateral neglect to a line bisection task and also asked them to estimate the body midline in the visual and proprioceptive modalities. Interestingly, to avoid any motor-exploratory component, the authors used the psychophysical method of constant stimuli, where steady stimuli are presented individually in predefined spatial locations and subjects are asked to judge whether they are located to the left or to the right of their objective sagittal middle. In accordance with our results and previous data, a significant rightward bias was found in the line bisection task, while no consistent directional bias was found in either the proprioceptive or visual body midline task.

This absence of correlation between performance in the proprioceptive, tactile and visual tasks does not necessarily imply that neglect is a modality-specific disorder, but it clearly counters any hypothesis of a causal relationship between the position of the ER and neglect signs in the tactile and visual modalities.

One possible explanation for the discrepancy between the straight-ahead pointing and the visual and tactile bisection performance could be that the tasks are not performed in the same spatial frame of reference. Whereas the straight-ahead pointing task would be performed in a body centered, ER, the rod and line bisection tasks would be performed in an object centered, allocentric frame of reference. The discrepancy between the two earlier mentioned frames of references could be enhanced by the nonbody-centered status of the hand [27]. However, the correlation found here between the proprioceptive straight ahead and rod bisection performances in normals argues against such a hypothesis, and suggests that, at least in normals, proprioceptive and tactile-kinesthetic estimations of the subjective middle share some common mechanisms; these latter could include the use of an egocentric, body-centered frame of reference. However, in accordance with previous findings $[1,11,13,22,47]$, even if left neglect is often observed in egocentric coordinates in most RBD patients, the body centered, ER could be distorted [49] but would not necessarily be shifted in a systematic ipsilesional way as postulated elsewhere $[36,38,39,43]$. It follows from these considerations that the transient remission of left neglect signs obtained with vestibular-proprioceptive experimental stimulations $[41,50,54,57,62]$ should perhaps not be interpreted in terms of a restoration of a sub-normal position of the ER but that these stimulations could act by allowing an orientation of attention to the left hemispace (see [3]).

In conclusion, the present findings argue in favor of an attentional bias in left neglect patients, that would take the form of both contralesional "hypoattention" and ipsilesional "hyperattention" that would be favored by the presence of surround stimuli in the right hemispace, as in the visual modality [2]. Thus, if neglect is linked more to an attentional bias than to a spatial distortion, a more systematic and severe rightward bias would be expected in the visual than in the proprioceptive and tactile modalities, as observed in the earlier mentioned studies. In this case, one should not necessarily expect to find a correlation between performance in the different modalities. More experiments are needed to test this hypothesis, including further studies of LBD patients with right unilateral spatial neglect.

\section{Acknowledgements}

A grant for this research was supported by the Rhône-Alpes region (contrat thématique) to the first and second authors. We wish to thank the CREA (Coubert) for the neglect patients referring, and the two anonymous reviewers.

\section{References}

[1] Bartolomeo P, Chokron S. Egocentric frame of reference: its role in spatial bias after right hemisphere lesions. Neuropsychologia 1999;37:881-94.

[2] Bartolomeo P, Chokron S. Left neglect or right hyper-attention? Neurology 1999;53:2023-7.

[3] Bartolomeo P, Chokron S. Levels of impairment in unilateral neglect. In: Boller F, Grafman J, editors. Disorders of visual behavior: handbook of neuropsychology. 2nd ed. Elsevier: Amsterdam, 2001. p. 67-98.

[4] Bartolomeo P, D'Erme P, Gainotti G. The relationship between visuospatial and representational neglect. Neurology 1994;44:1710 4.

[5] Battersby WS, Bender MB, Pollack M, Kahn RL. Unilateral "spatial agnosia" ("inattention") in patients with cerebral lesions. Brain 1956;79:68-93.

[6] Beschin N, Cubelli R, Della Sala S, Spinazzola L. Left of what? The role of egocentric coordinates in neglect. Journal of Neurology, Neurosurgery and Psychiatry 1997;63:483-9.

[7] Bisiach E, Luzzatti C. Unilateral neglect of representational space. Cortex 1978;14:129-33.

[8] Bowers D, Heilman KM. Pseudoneglect: effects of hemispace on a tactile line bisection task. Neuropsychologia 1980;18:491-8.

[9] Chedru F. Space representation in unilateral spatial neglect. Journal of Neurology, Neurosurgery and Psychiatry 1976;39:1057-61.

[10] Chokron S, Bartolomeo P. Correlation between the position of the egocentric reference and right neglect signs in left-brain damaged patients. Brain and Cognition 2000;43:99-104.

[11] Chokron S, Bartolomeo P. Patterns of dissociation between left hemineglect and deviation of the egocentric reference. Neuropsychologia 1997;35:1503-8. 
[12] Chokron S, Bartolomeo P. Pointing straight ahead: Reversed patterns of performance in right-brain damaged patients with or without extensive parietal lesion. Brain and Cognition 1999;40:79-84.

[13] Chokron S, Bartolomeo P. Position of the egocentric reference and directional arms movements in right-brain damaged patients. Brain and Cognition 1998;37:405-18.

[14] Chokron S, Bartolomeo P, Perenin MT, Helft G, Imbert M. Scanning direction and line bisection: a study of normal subjects and unilateral neglect patients with opposite reading habits. Cognitive Brain Research 1998;7:174-8.

[15] Chokron S, Imbert M. Egocentric reference and asymmetric perception of space. Neuropsychologia 1993;31:775-82.

[16] Chokron S, Imbert M. Variations of the egocentric reference among normal subjects and a patient with unilateral neglect. Neuropsychologia 1995;33:703-11.

[17] Chow SL. Précis of statistical significance: rationale, validity and utility. Behavioral and Brain Sciences 1998;21:169-239.

[18] Cohen J. Statistical power analysis for the behavioral sciences. New York: Academic Press, 1977.

[19] Corroyer D, Rouanet H. Sur l'importance des effets et ses indicateurs dans l'analyse statistique des données. L'Année Psychologique 1994;94:607-24.

[20] De Renzi E, Faglioni P, Scotti G. Hemispheric contribution to the exploration of space through the visual and tactile modality. Cortex 1970;6:191-203.

[21] Delatollas G, De Agostini M, Jallon P, Poncet M, Rey M, Lellouch J. Mesure de la préférence manuelle par auto-questionnaire dans la population française adulte. Revue de Psychologie Appliquée 1988;38:117-36

[22] Farne A, Ponti F, Ladavas E. In search for biased egocentric reference frames in neglect. Neuropsychologia 1998;36:611-23.

[23] Fujii T, Fukatsu R, Kimura I, Saso S-I, Kogure K. Unilateral spatial neglect in visual and tactile modalitites. Cortex 1991;27:339-43.

[24] Gainotti G. The role of spontaneous eye movements in orienting attention and in unilateral neglect. In: Robertson IH, Marshall JC, editors. Unilateral neglect: clinical and experimental studies. Hove (UK): Lawrence Erlbaum, 1993. p. 107-22.

[25] Gainotti G, D'Erme P, Bartolomeo P. Early orientation of attention toward the half space ipsilateral to the lesion in patients with unilateral brain damage. Journal of Neurology, Neurosurgery and Psychiatry 1991;54:1082-9.

[26] Gentilini M, Barbieri C, De Renzi E, Faglioni P. Space exploration with and without the aid of vision in hemisphere-damaged patients. Cortex 1989;25:643-51.

[27] Guerraz M, Luyat M, Poquin D, Ohlmann T. The role of neck afferents in subjective orientation in the visual and tactile sensory modalities. Acta otolaryngologica 2000;120:735-8.

[28] Hager F, Volz HP, Gaser C, Mentzel HJ, Kaiser WA, Sauer $\mathrm{H}$. Challenging the anterior attentional system with a continuous performance task: a functional magnetic resonance imaging approach. European Archives Psychiatry Clinical Neuroscience 1998;248:16170.

[29] Halligan P, Manning L, Marshall JC. Hemispheric activation vs. spatio-motor cueing in visual neglect: a case study. Neuropsychologia 1990;28:1043-51.

[30] Halligan P, Marshall JC, Wade DT. Visuo-spatial neglect: underlying factors and test sensitivity. Lancet 1989;14:908-10.

[31] Halligan PW, Marshall JC. How long is a piece of string ? A study of line bisection in a case of visual neglect. Cortex 1988;24:321-8.

[32] Heilman KM, Bowers D, Watson RT. Performance on hemispatial pointing task by patients with neglect syndrome. Neurology 1983;33: $661-4$.

[33] Heilman KM, Watson RT, Valenstein E. Neglect and related disorders. In: Heilman KM, Valenstein E, editors. Clinical neuropsychology. New York: Oxford University Press, 1993. p. 279-336.

[34] Hjaltason H, Caneman G, Tegner R. Visual and tactile rod bisection in unilateral neglect. Cortex 1993;29:588-93.
[35] Hjaltason H, Tegner R. Darkness improves line bisection in unilateral spatial neglect. Cortex 1992;28:353-8.

[36] Jeannerod M, Biguer B. The directional coding of reaching movements: a visuomotor conception of visuospatial neglect. In: Jeannerod M, editor. Neurophysiological and neuropsychological aspects of spatial neglect. Amsterdam: Elsevier, 1987. p. 87-113.

[37] Jewell G, McCourt ME. Pseudoneglect: a review and meta-analysis of performance factors in line bisection tasks. Neuropsychologia 2000;38:93-110.

[38] Karnath H-O. Disturbed coordinate transformation in the neural representation of space as the crucial mechanism leading to neglect. Neuropsychological Rehabilitation 1994;4(2):147-50.

[39] Karnath H-O. Neural encoding of space in egocentric coordinates? Evidence for and limits of a hypothesis derived from patients with parietal lesions and neglect. In: Thier P, Karnath H-O, editors. Parietal lobe contributions to orientation in 3D space. Heidelberg: Springer, 1997. p. 497-520.

[40] Karnath H-O. Optokinetic stimulation influences the disturbed perception of body orientation in spatial neglect. Journal of Clinical and Experimental Neuropsychology 1996;60:217-20.

[41] Karnath HO. Subjective body orientation in neglect and the interactive contribution of neck muscle proprioception and vestibular stimulation. Brain 1994;117:1001-12.

[42] Karnath HO, Niemer M, Dichgans J. Space exploration in neglect. Brain 1998;121:2357-67.

[43] Karnath H-O, Perenin MT. Tactile exploration of peripersonal space in patients with neglect. Neuropreport 1998;9:2273-7.

[44] Karnath H-O, Schenkel P, Fischer B. Trunk orientation as the determining factor of the contralateral deficit in the neglect syndrome and as the physical anchor of the internal representation of body orientation in space. Brain 1991;114:1997-2014.

[45] Kinsbourne M. Mechanisms of unilateral neglect. In: Jeannerod M, editor. Neurophysiological and neuropsychological aspects of spatial neglect. Amsterdam: Elsevier, 1987. p. 69-86.

[46] Mattingley JB, Pierson JM, Bradshaw JL, Phillips JG, Bradshaw JA. To see or not to see: the effect of visible and invisible cues on line bisection judgments in unilateral neglect. Neuropsychologia 1993;31:1201-15.

[47] Perenin MT. Optic ataxia and unilateral neglect: clinical evidence for dissociable spatial functions in posterior parietal cortex. In: Thier P, Karnath HO, editors. Parietal lobe contributions to orientation in 3D space. Heidelberg: Springer, 1997. p. 289-308.

[48] Philip J, Hatwell Y. Effects of cueing and of the direction of scanning on the tactile line bisection of normal adults. CPC Current Psychology on Cognition 1998;17:31-51.

[49] Pizzamiglio L, Committeri G, Galati G, Patria F. Psychophysical properties of line bisection and body midline perception in unilateral neglect. Cortex 2000;36:469-84.

[50] Pizzamiglio L, Frasca R, Guariglia C, Incoccia C, Antonucci G. Effect of optokinetic stimulation in patients with visual neglect. Cortex 1990;26:535-40.

[51] Posner MI. Orienting of attention. Quarterly Journal of Experimental Psychology 1980;32:3-25.

[52] Posner MI, Walker JA, Friedrich FJ, Rafal RD. Effects of parietal injury on covert orienting of attention. Journal of Neuroscience 1984;4:1863-4.

[53] Reuter-Lorenz PA, Posner MI. Components of neglect from righthemisphere damage: an analysis of line bisection. Neuropsychologia 1990;28(4):327-33.

[54] Rode G, Perenin MT. Temporary remission of representational hemineglect through vestibular stimulation. Neuroreport 1994;5:86972.

[55] Rode G, Perenin MT, Boisson D. Neglect of the physical space. Annales de réadaptation et de médecine physique 1996;39:165-9.

[56] Rosenthal R, Rubin DB. The countermull value of an effect size: a new statistic. Psychological Science 1994;5:329-34. 
[57] Rossetti Y, Rode G, Pisella L, Farne A, Li L, Boisson D. Prism adaptation to a rightward optical deviation rehabilitates left hemispatial neglect. Nature 1998;395:166-9.

[58] Sampaio E, Chokron S. Pseudoneglect and reversed pseudoneglect among left-handers and right-handers. Neuropsychologia 1992;30: 797-805.

[59] Sampaio E, Philip J. Sensory and motor aspects of pseudoneglect, hemifield and hemiespace in the tactile modality. Brain and Cognition 1991;16:74-82.

[60] Tegnèr R, Levander M, Caneman G. Apparent right neglect in patients with left visual neglect. Cortex 1990;26:455-8.

[61] Turvey MT. Dynamic touch. American Psychologist 1996;51:113452.

[62] Vallar G, Antonucci G, Guariglia C, Pizzamiglio L. Deficits of position sense, unilateral neglect and optokinetic stimulation. Neuropsychologia 1993;31:1191-200.
[63] Vallar G, Guariglia C, Nico D, Bisiach E. Spatial hemineglect in back space. Brain 1995;118:467-72.

[64] Vallar G, Guariglia C, Rusconi ML. Modulation of neglect syndrome by sensory stimulation. In: Thier P, Karnath H-O, editors. Parietal lobe contributions to orientation in 3D space. Heidelberg: Springer, 1997.

[65] Vallar G, Rusconi ML, Barozzi S, Bernardini B, Ovadia D, Papagno $\mathrm{C}$, et al. Improvement of left visuo-spatial hemineglect by left-sided transcutaneous electrical stimulation. Neuropsychologia 1995;33:7382.

[66] Villardita C. Tactile exploration of space and visual neglect in brain-damaged patients. Journal of Neurology 1987;234:2927.

[67] Weintraub S, Mesulam MM. Visual hemispatial inattention: stimulus parameters and exploratory strategies. Journal of Neurology, Neurosurgery and Psychiatry 1988;51:1481-8. 